\title{
In vitro and in vivo characterization of a cranial window prosthesis for diagnostic and therapeutic cerebral ultrasound
}

\author{
Francesco Prada, MD, ${ }^{1-3}$ Andrea Franzini, MD, ${ }^{1,2}$ Shayan Moosa, MD, ${ }^{2}$ Frederic Padilla, PhD, ${ }^{3}$ \\ David Moore, MS, ${ }^{3}$ Luigi Solbiati, MD, ${ }^{4}$ Francesco DiMeco, MD, ${ }^{1,5,6}$ and Wynn Legon, ${ }^{2 h D}{ }^{2}$

\begin{abstract}
${ }^{1}$ Department of Neurosurgery, Fondazione IRCCS Istituto Neurologico C. Besta, Milano, Italy; ${ }^{2}$ Department of Neurosurgery, University of Virginia Health System; ${ }^{3}$ Focused Ultrasound Foundation, Charlottesville, Virginia; ${ }^{4}$ Department of Radiology, Humanitas Research Hospital, Rozzano, Italy; ${ }^{5}$ Department of Neurosurgery, Johns Hopkins University, Baltimore, Maryland; and ${ }^{6}$ Department of Pathophysiology and Transplantation, Università degli studi di Milano, Italy
\end{abstract}

\begin{abstract}
OBJECTIVE The authors evaluated the acoustic properties of an implantable, biocompatible, polyolefin-based cranial prosthesis as a medium to transmit ultrasound energy into the intracranial space with minimal distortion for imaging and therapeutic purposes.

METHODS The authors performed in vitro and in vivo studies of ultrasound transmission through a cranial prosthesis. In the in vitro phase, they analyzed the transmission of ultrasound energy through the prosthesis in a water tank using various transducers with resonance frequencies corresponding to those of devices used for neurosurgical imaging and therapeutic purposes. Four distinct, single-element, focused transducers were tested at fundamental frequencies of 500 $\mathrm{kHz}, 1 \mathrm{MHz}, 2.5 \mathrm{MHz}$, and $5 \mathrm{MHz}$. In addition, the authors tested ultrasound transmission through the prosthesis using a linear diagnostic probe (center frequency $5.3 \mathrm{MHz}$ ) with a calibrated needle hydrophone in free water. Each transducer was assessed across a range of input voltages that encompassed their full minimum to maximum range without waveform distortion. They also tested the effect of the prosthesis on beam pressure and geometry. In the in vivo phase, the authors performed ultrasound imaging through the prosthesis implanted in a swine model.
\end{abstract}

RESULTS Acoustic power attenuation through the prosthesis was considerably lower than that reported to occur through the native cranial bone. Increasing the frequency of the transducer augmented the degree of acoustic power loss. The degradation/distortion of the ultrasound beams passing through the prosthesis was minimal in all 3 spatial planes ( $X Y, X Z$, and $Y Z$ ) that were examined. The images acquired in vivo demonstrated no spatial distortion from the prosthesis, with spatial relationships that were superimposable to those acquired through the dura.

CONCLUSIONS The results of the tests performed on the polyolefin-based cranial prosthesis indicated that this is a valid medium for delivering both focused and unfocused ultrasound and obtaining ultrasound images of the intracranial space. The prosthesis may serve for several diagnostic and therapeutic ultrasound-based applications, including bedside imaging of the brain and ultrasound-guided focused ultrasound cerebral procedures.

https://thejns.org/doi/abs/10.3171/2019.10.JNS191674

KEYWORDS prosthesis; cranial window; neurosurgery; focused ultrasound; brain imaging; diagnostic technique

$\mathrm{D}$ IAGNOSTIC and therapeutic ultrasound (US) technologies have a multitude of uses in the management of neurological disorders. However, the human skull reflects or absorbs a large amount of ultrasonic energy, ${ }^{3,33,35}$ which significantly hampers acoustic transmission between external transducers and intracranial structures. ${ }^{15,33}$ Experimental studies have demonstrated that the transmission of US beams through the skull is extremely low. ${ }^{12}$ Also, focusing US for therapeutic purposes requires complex corrections of phase distortions of the US beams that are caused by the skull. ${ }^{8}$ To improve the passage of US to the brain, the presence of an acoustic window would be extremely advantageous from both a diagnostic and a therapeutic standpoint. Acoustic windows

ABBREVIATIONS BBB = blood-brain barrier; FWHM = full width half maximum; PMMA = polymethyl methacrylate; US = ultrasound.

SUBMITTED June 16, 2019. ACCEPTED October 28, 2019.

INCLUDE WHEN CITING Published online January 3, 2020; DOI: 10.3171/2019.10.JNS191674. 
are naturally present in the adult human skull, where bone is thinner, such as the temporal bone area, but they are limited in their extension and permit only a narrow and degraded view of intracranial structures. Therefore, some authors have sought to replace part of the skull with materials that are more permeable to acoustic energy., ${ }^{4,43}$ Various materials have been tested for this purpose, including multiple plastics and ceramics. ${ }^{4,15,34,43}$ In these studies, materials were tested mostly for delivering US with therapeutic aims, such as for the ablation of intracranial tumors, whereas intracranial diagnostic imaging applications have had little consideration.

In the present study, we use an implantable biocompatible polyolefin-based prosthesis (TM, In.Tra.) as a medium to transmit US into the intracranial space with minimal distortion for imaging and therapeutic purposes.

In the first phase of the study, we tested the propagation of US in vitro through the prosthesis by using various US transducers. In the second phase, in order to validate the prosthesis for intracranial imaging, we implanted the prosthesis in a swine model (Sus scrofa domesticus), which, due to its size and large gyrated brain, is a well-suited model for human brain research ${ }^{28}$ and can be conveniently imaged with conventional human US transducers. Finally, we discuss the possible future diagnostic and therapeutic neurosurgical applications of such an acoustically permeable prosthesis.

\section{Methods \\ Study Design}

A 2-phase study was conducted in an effort to analyze in vitro the transmission of US through a sonolucent biocompatible cranial prosthesis made of a polyolefin-based material, and to investigate in vivo whether it may be a useful window for visualizing the intracranial space with current US technologies. The first phase of the study was conducted in a water tank to test the acoustic impedance of the prosthesis with various US transducers registered with a needle hydrophone, whereas the second phase was aimed at testing US imaging in vivo using a swine model.

\section{Biocompatible Cranial Prosthesis Properties}

In vitro power transmission tests and in vivo imaging were performed on sterilizable, 4-mm-thick, $3 \times 6-\mathrm{cm}$ and $4 \times 4-\mathrm{cm}$ pieces of polyolefin polymers. The material was produced by machining. The density and porosity of the materials were $0.93 / \mathrm{cm}^{3}$ and $0.44 \mathrm{~g} / \mathrm{cm}^{3}$, respectively.

\section{Phase 1: In Vitro Acoustic Analysis (Water Tank) \\ Empirical Acoustic Field Mapping}

We measured the characteristics of each transducer in free water and through a $3 \times 6-\mathrm{cm}, 4-\mathrm{mm}$-thick polyolefin-based polymer prosthesis in an acoustic test tank filled with deionized, degassed, and filtered water (Precision Acoustics Ltd.). A calibrated hydrophone (HNR-0500, Onda Corp.) mounted on a motorized stage was used to measure the pressure profile from the US transducer (Fig. 1A). All scans were performed at $100-\mu \mathrm{m}$ spatial resolution. Waveforms were generated using a function genera- tor (AFG 3022C, Tektronix) and fed into a class A, 100-W, 50-dB linear amplifier (2100 L, E\&I) that powered the transducers. Waveforms were viewed and stored using an oscilloscope (DS0 × 3012T, Keysight Technologies). All acquisition was conducted and controlled by an US measuring system (Precision Acoustics Ltd.) (Fig. 1A). Data from the acquisition system were analyzed using custom scripts written in MATLAB (MathWorks). We collected data from 4 focused, single-element transducers $(500 \mathrm{kHz}$, $1 \mathrm{MHz}, 2.5 \mathrm{MHz}$, and $5 \mathrm{MHz}$ ), as well as a clinical scanner (ECO6 US system; 5.3-MHz linear probe L7M [Chison]). The $500-\mathrm{kHz}$ transducer was a custom-made $\varnothing 30$ $\mathrm{mm}$ focused transducer that we have used in previous neuromodulation studies. ${ }^{1,25-27}$ The $1-\mathrm{MHz}$ transducer was an H-102 1.1-MHz, Ø64-mm, 63.5-mm focused transducer (Sonic Concepts); the 2.5-MHz transducer was an $\mathrm{H}-111$ 2.5-MHz, Ø25-mm, 35-mm focused transducer (Sonic Concepts); and the 5-MHz transducer was a V310-N-SU 5-MHz, Ø6.35-mm, 20-mm focused transducer (Olympus Corp.). For each transducer, prior to formal data collection, the US beam maximum was searched for in free water. We then conducted $\mathrm{X}, \mathrm{Y}$, and $\mathrm{Z}$ linear scans, a voltage sweep, and a planer XY scan in free water. For $Z$ linear scans using the $500-\mathrm{kHz}, 2.5-\mathrm{MHz}$, and $5-\mathrm{MHz}$ transducers, the hydrophone was brought to a point $5 \mathrm{~mm}$ from the exit plane of the transducer to allow for the same $\mathrm{Z}$ scan when the prosthesis was placed between the exit plane and the hydrophone (Fig. 1B). This was not possible for the $1-\mathrm{MHz}$ test as the diameter of the transducer was larger than the prosthesis and did not permit this configuration. Instead, a coupling cone with an offset of $35 \mathrm{~mm}$ (that corresponded to the focal length) from the exit plane was used and was able to be placed on the prosthesis. In all cases, free-water scans were acquired prior to the prosthesis scans, and no parameters were changed between the free-water scan and the prosthesis scan. All free-water and prosthesis scans for each transducer were collected on the same day in consecutive order. After all free-water scans, the prosthesis was carefully positioned $3 \mathrm{~mm}$ in the $\mathrm{Z}$ axis from the exit plane of the transducer and centered in X and Y planes. All X, Y, and $\mathrm{Z}$ linear scans and the $\mathrm{XY}$ planar scan were obtained at a $50-\mathrm{mVpp}$ input voltage except for the $2.5-\mathrm{MHz}$ transducer test, which was collected at $20 \mathrm{mVpp}$.

\section{Phase 2: In Vivo Swine Model}

\section{Study Design}

This part of the study was approved by the Institutional Care and Animal Use Committee at the University of Virginia. Procedures were performed in the University of Virginia animal surgery suite. Two female Yorkshire swine (Sus scrofa domesticus) weighing 25-30 pounds and being 6-7 weeks of age were used in this experiment. We performed intraoperative US evaluation of healthy pig brains following a wide frontoparietal craniectomy procedure. US assessment was carried out in two different conditions. At first, the US diagnostic probe was held on the intact dura. Next, a polyolefin-based cranial prosthesis was placed in the cranial defect, and images were acquired through the prosthesis, which therefore served as an acoustic window (Fig. 2A and B). 

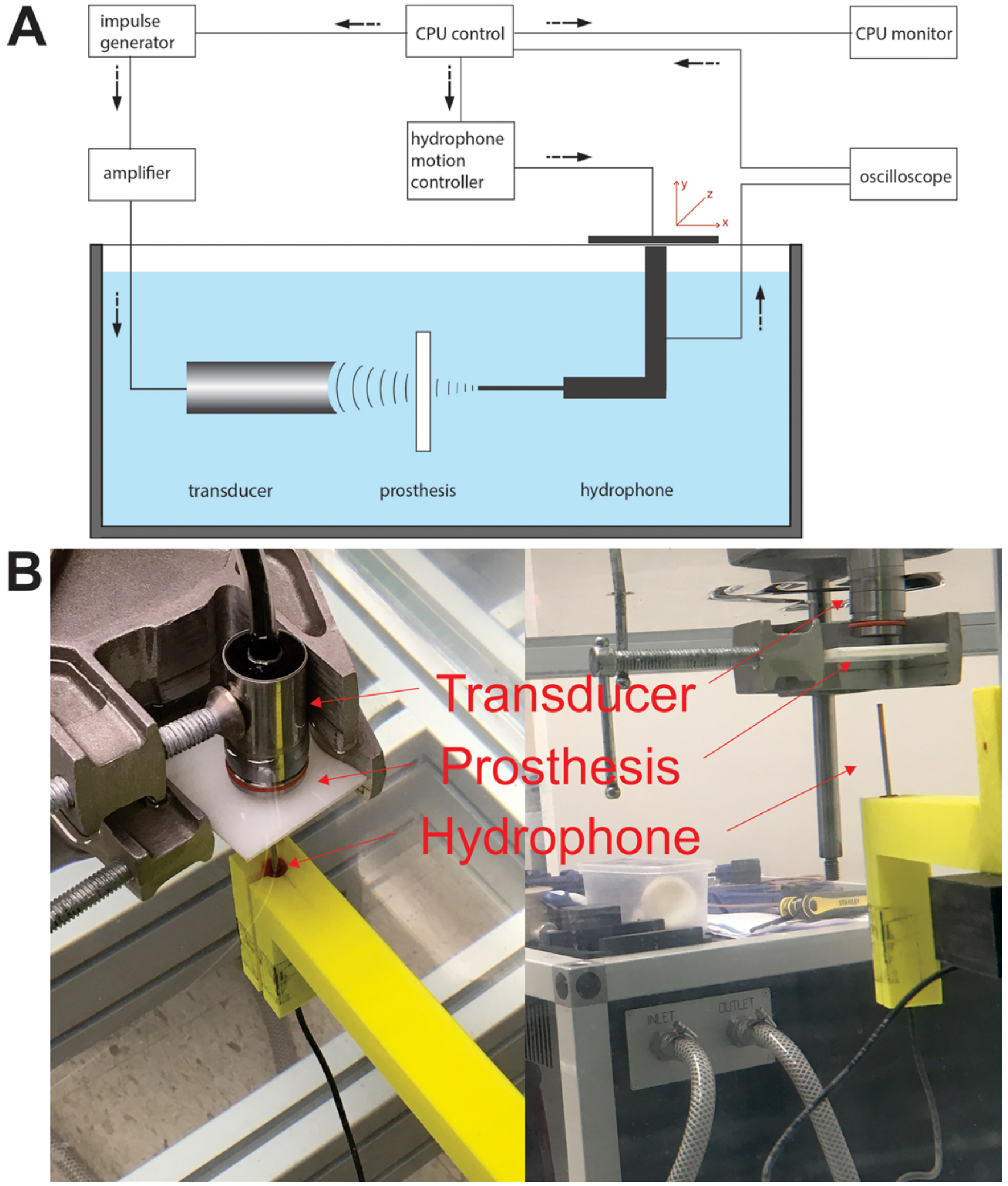

FIG. 1. A: Schematic of the recording setup. B: Photographs showing the relative position of the transducer (2.5 MHz), prosthesis (white), and hydrophone. Note that there is slight distortion in the photograph on the right side due to the refraction of light in water. Figure is available in color online only. 

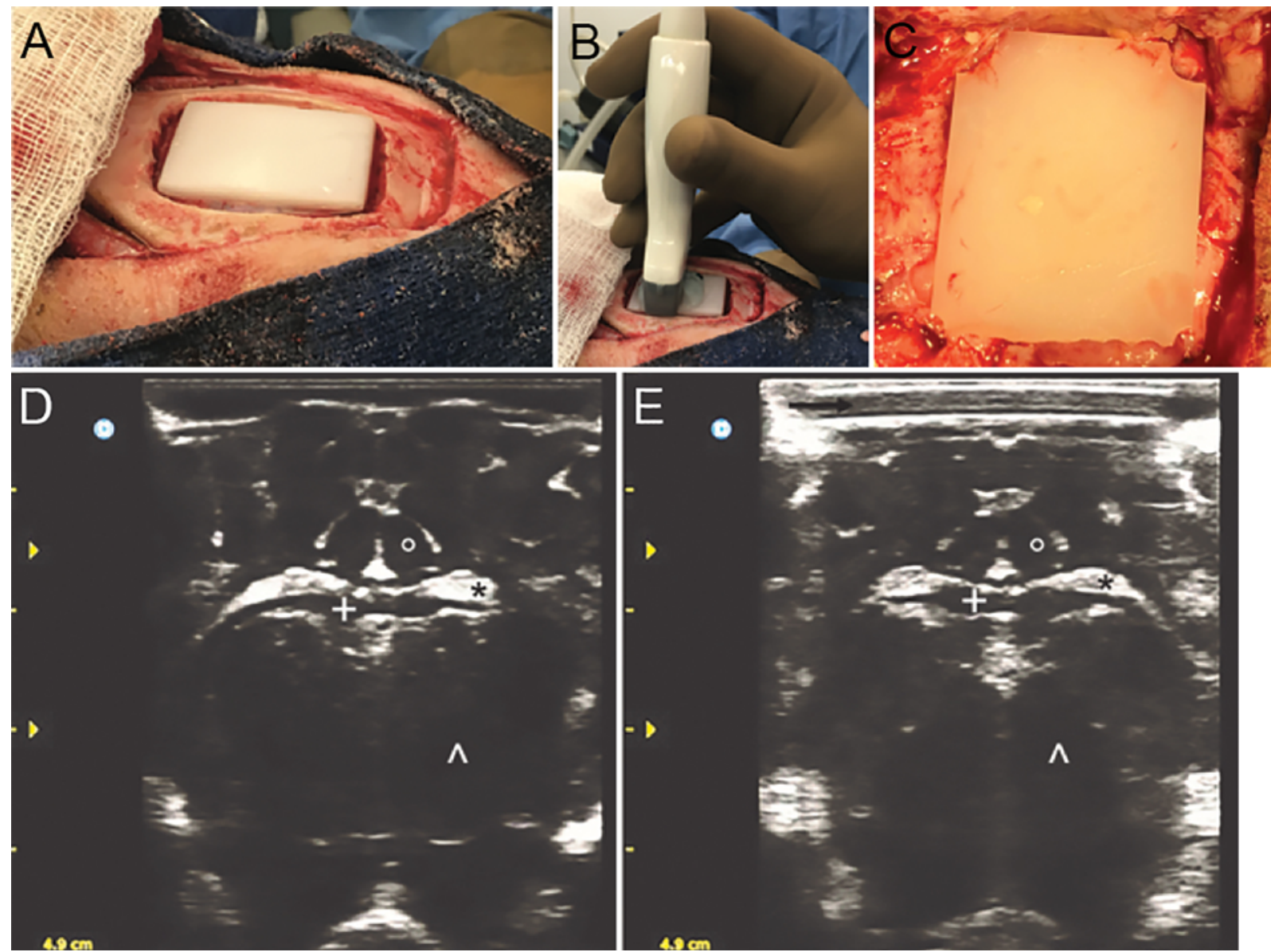

FIG. 2. A: The prosthesis is placed in the bony window created using a wide frontoparietal craniectomy. B: Sonographic images are obtained through the prosthesis and sonographic gel. C: In this pig, the prosthesis was removed at 8 weeks after implantation. No significant bony overgrowth or periprosthetic scar was evident. D and E: Sonographic images of the swine's brain obtained through the meninges (D) and the prosthesis (E). The thalamus $\left({ }^{\wedge}\right)$, cingulate gyrus (empty circle), lateral ventricle (asterisk), and the corpus callosum (cross) are readily identifiable on both images. In these sonographic images ( $D$ and $E$ ), the prosthesis was free floating $(B)$ or fashioned to fix precisely in the cranial defect to eliminate movement $(C)$. We envision that it would be possible to fix the prosthesis to the surrounding natural bone with nonabsorbable sutures or titanium miniplates and screws, which can be easily inserted into the prosthesis. Figure is available in color online only.

\section{Anesthesia and Surgery}

Prior to treatment, the pigs were sedated with a single intramuscular injection of tiletamine (Telazol $6 \mathrm{mg}$ / $\mathrm{kg})$ mixed with xylazine $(2.2 \mathrm{mg} / \mathrm{kg})$, followed by endotracheal intubation. Anesthesia was maintained with a continuous infusion of propofol at $10 \mathrm{mg} / \mathrm{kg} / \mathrm{hr}$. Animals were placed on a ventilator (10-ml $/ \mathrm{kg}$ tidal volume) at a rate of 18 breaths $/ \mathrm{min}$. An intravenous catheter was placed in the marginal vein of each ear. Vital signs were monitored throughout the entirety of the procedure. After anesthetization, a U-shaped incision was made in order to reflect the scalp posteriorly, and a $4 \times 4-\mathrm{cm}$ craniectomy (matching the dimensions of the prosthesis) centered on the bregma was performed with a high-speed drill in order to create space for the prosthesis. Bone removal involved the flat surface of the parietal and frontal bone bilaterally, so as to allow imaging of the ventricles and of the diencephalic structures in each pig. The dura was kept intact. Thereafter, the cranial prosthesis was placed in the bony window.

\section{Ultrasound Evaluation}

For US imaging, we used an US system equipped with a multifrequency linear probe (ECO6 US system; 2- to 7-MHz linear probe L7M [Chison]). At first, the probe was placed over the external surface of the dura to acquire baseline bidimensional-mode (B-mode) images in both coronal and sagittal orientations. A frequency of 5.3 $\mathrm{MHz}$ was chosen to image the brain, as this allowed us to obtain good anatomical detail with sufficient depth imaging (maximum depth $4.9 \mathrm{~cm}$ ). With this frequency, a morphological qualitative analysis of the swine brain anatomy was performed. Two-dimensional images of the cortex, ventricles, and subcortical brain regions were acquired. A second imaging session was performed after the prosthesis had been placed into the bone defect (Fig. 2D and 

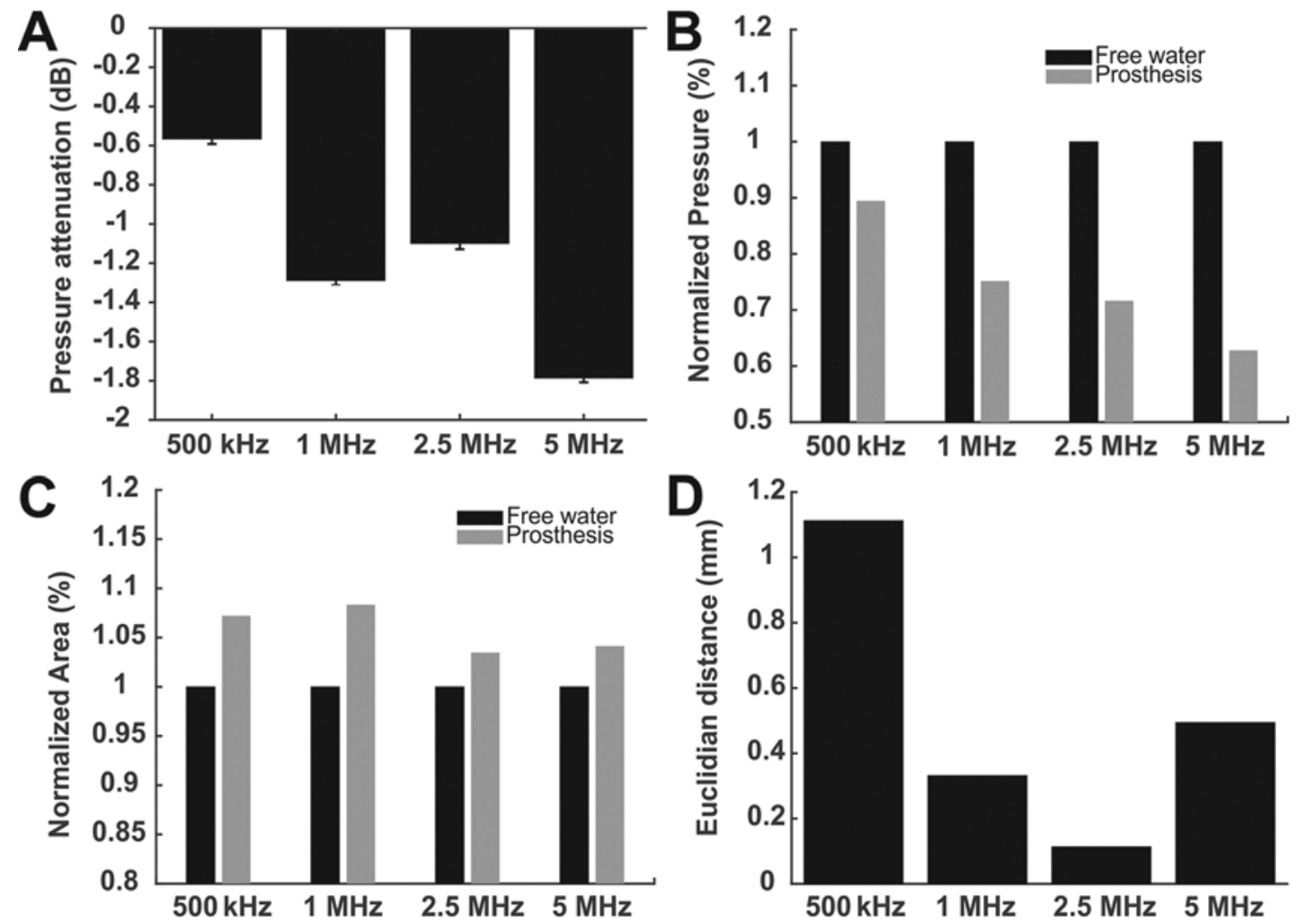

FIG. 3. A: Average pressure attenuation in decibels through the prosthesis as compared to free water across the full input voltage range. B: Normalized pressure attenuation at maximum input voltage. C: FWHM cross-sectional XY pressure area at Z focus normalized to free-water values (= 1). D: Absolute translation of centroid of FWHM pressure area from data in $\mathrm{C}$ through the prosthesis as compared to free-water measurements. Negative values represent being closer to the transducer exit plane.

E). The same frequency of the previous imaging session (5.3 MHz) was chosen, and images corresponding to those of the baseline session were acquired by orienting the US probe in the same planes and directions. Subsequently, the two image sets were compared to detect qualitative differences. Following surgery and US evaluation, the pigs were euthanized with intravenously administered pentobarbital $(140-160 \mathrm{mg} / \mathrm{kg})$.

\section{Results}

\section{Preclinical Acoustic Analysis}

We tested 4 different single-element focused transducers at fundamental frequencies of $500 \mathrm{kHz}, 1 \mathrm{MHz}, 2.5$ $\mathrm{MHz}$, and $5 \mathrm{MHz}$, as well as a linear diagnostic probe (center frequency $5.3 \mathrm{MHz}$ ) with a calibrated needle hydrophone, in free water and through a $4 \times 4-\mathrm{cm}$ and 4-mm-thick polyolefin prosthesis. We tested the effects of the prosthesis on pressure and beam geometry. For all transducers tested, the prosthesis resulted in a decrease in maximum pressure. Pressure attenuation at maximum input voltage was $-0.484 \mathrm{~dB},-1.239 \mathrm{~dB},-1.448 \mathrm{~dB}$, and $-2.02 \mathrm{~dB}$ for the $500 \mathrm{kHz}, 1 \mathrm{MHz}, 2.5 \mathrm{MHz}$, and $5 \mathrm{MHz}$ transducers, respectively (Fig. 3A, Table 1). We tested each transducer across a range of input voltages that encompassed their full minimum to maximum range without waveform distortion. The mean attenuation across this range was $-0.567 \pm 0.023 \mathrm{~dB},-1.29 \mathrm{~dB} \pm 0.02 \mathrm{~dB},-1.1 \mathrm{~dB}$ $\pm 0.03 \mathrm{~dB}$, and $-1.79 \pm 0.02 \mathrm{~dB}$ for $500 \mathrm{kHz}, 1 \mathrm{MHz}, 2.5$ $\mathrm{MHz}$, and $5 \mathrm{MHz}$, respectively (Figs. 3B, 4-6, and 7A-C, Table 1). The low variability demonstrates that the attenu-

TABLE 1. Pressures and attenuation for the various frequency transducers tested

\begin{tabular}{ccccc}
\hline Frequency & $\begin{array}{c}\text { Free Water } \\
(\mathrm{kPa})\end{array}$ & $\begin{array}{c}\text { Prosthesis } \\
(\mathrm{kPa})\end{array}$ & $\begin{array}{c}\text { Difference } \\
(\mathrm{kPa})\end{array}$ & $\mathrm{dB}$ \\
\hline Maximum input voltages & \multicolumn{4}{l}{} \\
\hline $500 \mathrm{kHz}$ & 794.5 & 710.7 & 83.79 & -0.484 \\
\hline $1 \mathrm{MHz}$ & 5256.3 & 3951.1 & 1305.2 & -1.239 \\
\hline $2.5 \mathrm{MHz}$ & 3256 & 2332.6 & 923.48 & -1.448 \\
\hline $5 \mathrm{MHz}$ & 141.28 & 88.73 & 52.55 & -2.02 \\
\hline Average input voltages from full minimum to maximum range \\
\hline $500 \mathrm{kHz}$ & 381.76 & 335.17 & 46.59 & -0.567 \\
\hline $1 \mathrm{MHz}$ & 1297.9 & 964.86 & 333.04 & -1.29 \\
\hline $2.5 \mathrm{MHz}$ & 1019.8 & 791.85 & 227.95 & -1.102 \\
\hline $5 \mathrm{MHz}$ & 172.1 & 114.06 & 58.04 & -1.789 \\
\hline
\end{tabular}

Values were recorded at the maximum input voltage and as an average of a range of input voltages that encompassed their full minimum to maximum range. 

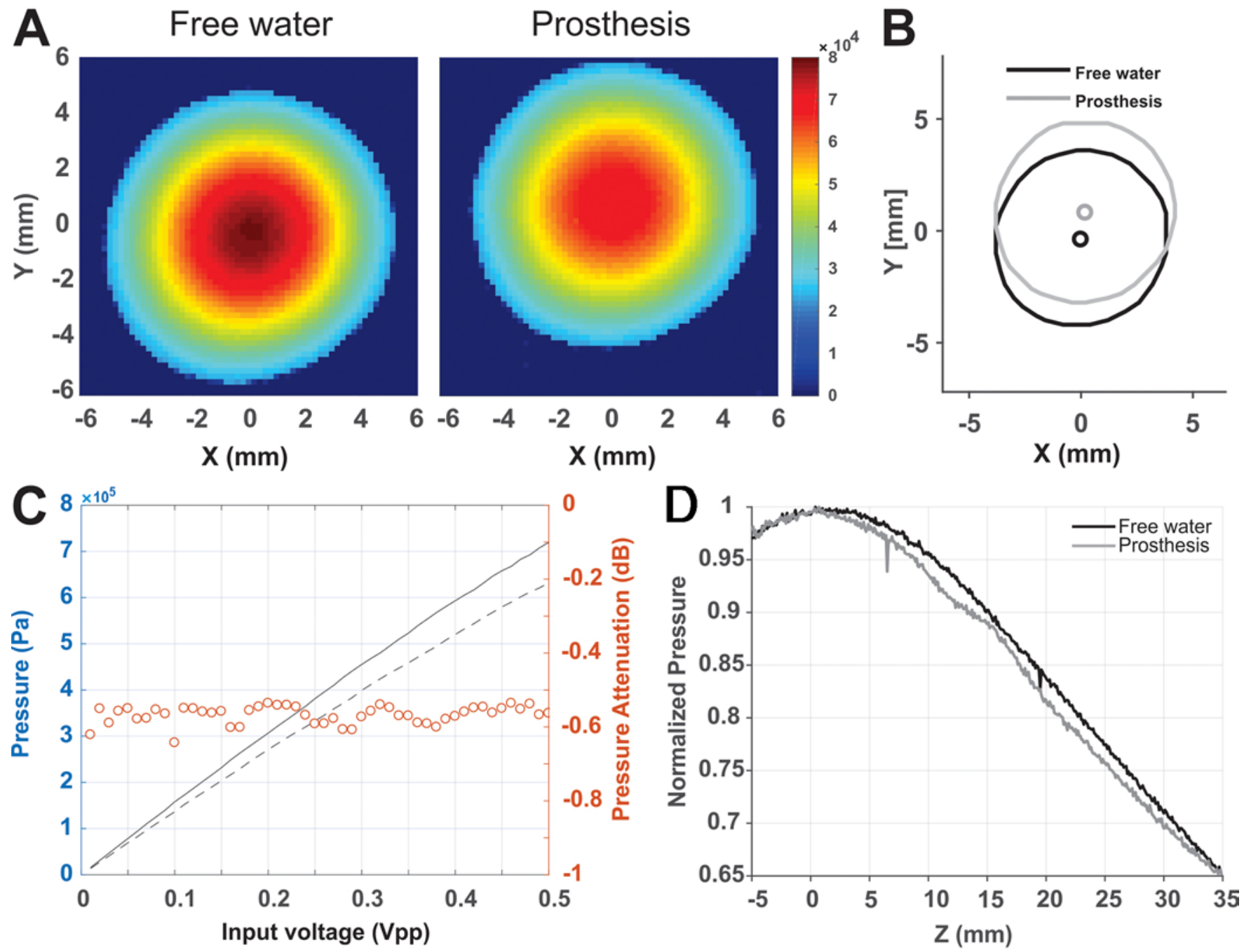

FIG. 4. Data for the 500-kHz transducer. A: XY cross-sectional pseudocolor pressure profiles at Z maximum. B: FWHM outline of the pressure map (large circles) and corresponding centroid (small circles). C: YY plot showing pressure in pascals (blue values) and attenuation through the prosthesis as compared to free water in decibels (red values) as a function of input voltage. The solid line is the recording in free water and the dashed line is the recording through the prosthesis. D: Pressure scan in Z access normalized to peak pressure. Zero on the $X$ axis denotes a pressure peak in the $Z$ plane found in free water. Negative values represent being closer to the transducer exit plane. Figure is available in color online only.

ation is roughly linear and stable across increasing input voltages up to the maximum for each transducer (Figs. 4C, $5 \mathrm{C}, 6 \mathrm{C}$, and $7 \mathrm{C}$ ). We also tested the effect of the prosthesis on the US beam geometry, including full width half maximum (FWHM) XY cross-sectional area, beam centroid translation, and $\mathrm{Z}$ axis focal length at a single input voltage. We found the prosthesis to increase the FWHM crosssectional area for all transducers (Fig. 3C). Increases were, respectively, for $500 \mathrm{kHz}, 47.23$ versus $50.64 \mathrm{~cm}^{2}$ (7.2\%); for $1 \mathrm{MHz}, 3.82$ versus $4.14 \mathrm{~cm}^{2}(8.4 \%)$; for $2.5 \mathrm{MHz}, 1.55$ versus $1.60 \mathrm{~cm}^{2}(3.23 \%)$; and for $5 \mathrm{MHz}, 7.84$ versus 8.17 $\mathrm{cm}^{2}(4.2 \%)$ (Figs. 3-5 and 6A and B). The translation of the centroid of the beam in the XY plane was $1.112 \mathrm{~mm}$, $0.332 \mathrm{~mm}, 0.114 \mathrm{~mm}$, and $0.494 \mathrm{~mm}$ for $500 \mathrm{kHz}, 1 \mathrm{MHz}$, $2.5 \mathrm{MHz}$, and $5 \mathrm{MHz}$, respectively (Figs. 3D, 4-6, and $7 \mathrm{~A}$ and $\mathrm{B})$. Finally, we performed line scans in the $\mathrm{Z}$ axis and found the prosthesis to translate the $\mathrm{Z}$ axis location of maximum pressure $0 \mathrm{~mm},-3.5 \mathrm{~mm},-3.0 \mathrm{~mm}$, and -2.0 $\mathrm{mm}$ for the $500-\mathrm{kHz}, 1-\mathrm{MHz}, 2.5-\mathrm{MHz}$, and $5-\mathrm{MHz}$ transducer, respectively (Figs. 4-6 and 7D).

\section{Clinical Transducer}

For the linear clinical probe, we measured the XY pressure map at the indicated focal length and a $\mathrm{Z}$ line scan to assess how the prosthesis affects the area and $\mathrm{Z}$ axis pressure profile. We found that the prosthesis resulted in a decrease in area of the FWHM of the pressure map at the $\mathrm{Z}$ focus from $404.85 \mathrm{~mm}^{2}$ to $206.45 \mathrm{~mm}^{2}$ (Fig. 8A and B). Interestingly, the pressure profile in the $\mathrm{Z}$ axis was considerably different through the prosthesis as compared to free water, where the pressure increased about $5 \mathrm{~mm}$ in the $\mathrm{Z}$ axis around for the prosthesis scan relative to the free- 

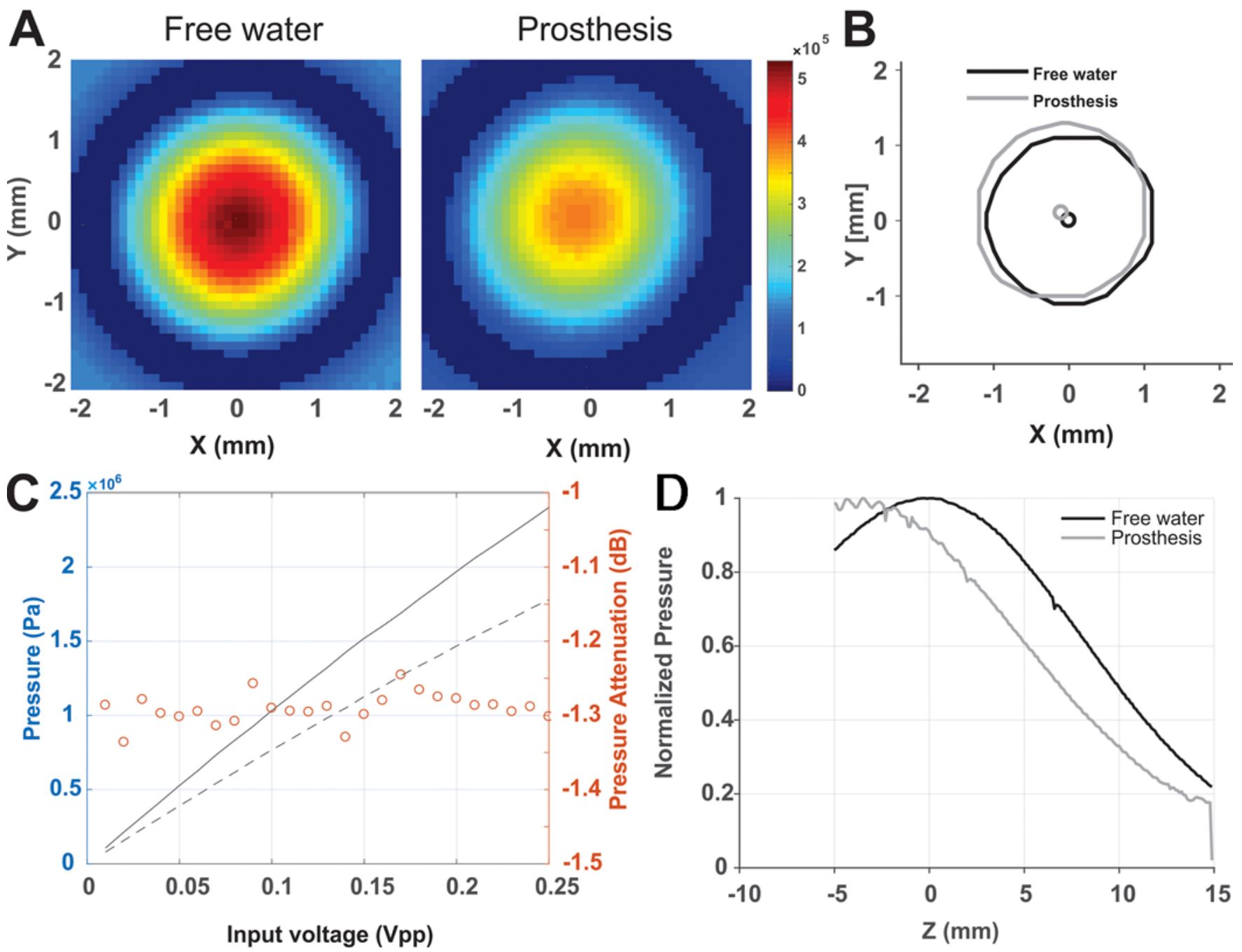

FIG. 5. Data for the 1-MHz transducer. A: XY cross sectional pseudocolor pressure profiles at Z maximum. B: FWHM outline of the pressure map (large circles) and corresponding centroid (small circles). C: YY plot showing pressure in pascals (blue values) and attenuation through the prosthesis as compared to free water in decibels (red values) as a function of input voltage. The solid line is the recording in free water and the dashed line is the recording through the prosthesis. D: Pressure scan in Z access normalized to peak pressure. Zero on the $X$ axis denotes a pressure peak in the $Z$ plane found in free water. Negative values represent being closer to the transducer exit plane. Figure is available in color online only.

water scan (Fig. 8D). This is evident in the pseudocolor $\mathrm{XY}$ (Fig. 8A and B), where the pressure is more uniform across the $\mathrm{Y}$ axis for the prosthesis as compared to the free water that displays peak pressures at the ends of the $\mathrm{Y}$ axis only. As tested here, the prosthesis appears to shorten the field in the $\mathrm{X}$ axis, which creates a more uniform pressure profile and smaller FWHM area.

\section{In Vivo Ultrasound Imaging}

We obtained B-mode US images in all cases after the craniectomy, both with direct transdural insonation and through the prosthesis serving as an acoustic window. The images were obtained in the same coronal plane for direct comparison and were evaluated by two expert sonographers (F. Prada and F. Padilla). Comparing transdural and transprosthesis imaging, a considerable anatomical correlation was found, with no distortion in terms of depth and structure echogenicity and with complete correspondence of anatomical structure location (Fig. 2D and E). The resolutions of the images obtained in both conditions were comparable. In the images obtained with the prosthesis placed in the cranial defect, we observed a small degree of lateral attenuation in the most peripheral areas of the ultrasonic field.

\section{Discussion}

The results of our study show that US can be effectively transmitted through a polyolefin-based cranial prosthesis, which may be implanted for both US-based diagnostic imaging and therapeutic purposes. In vitro, we found low at- 

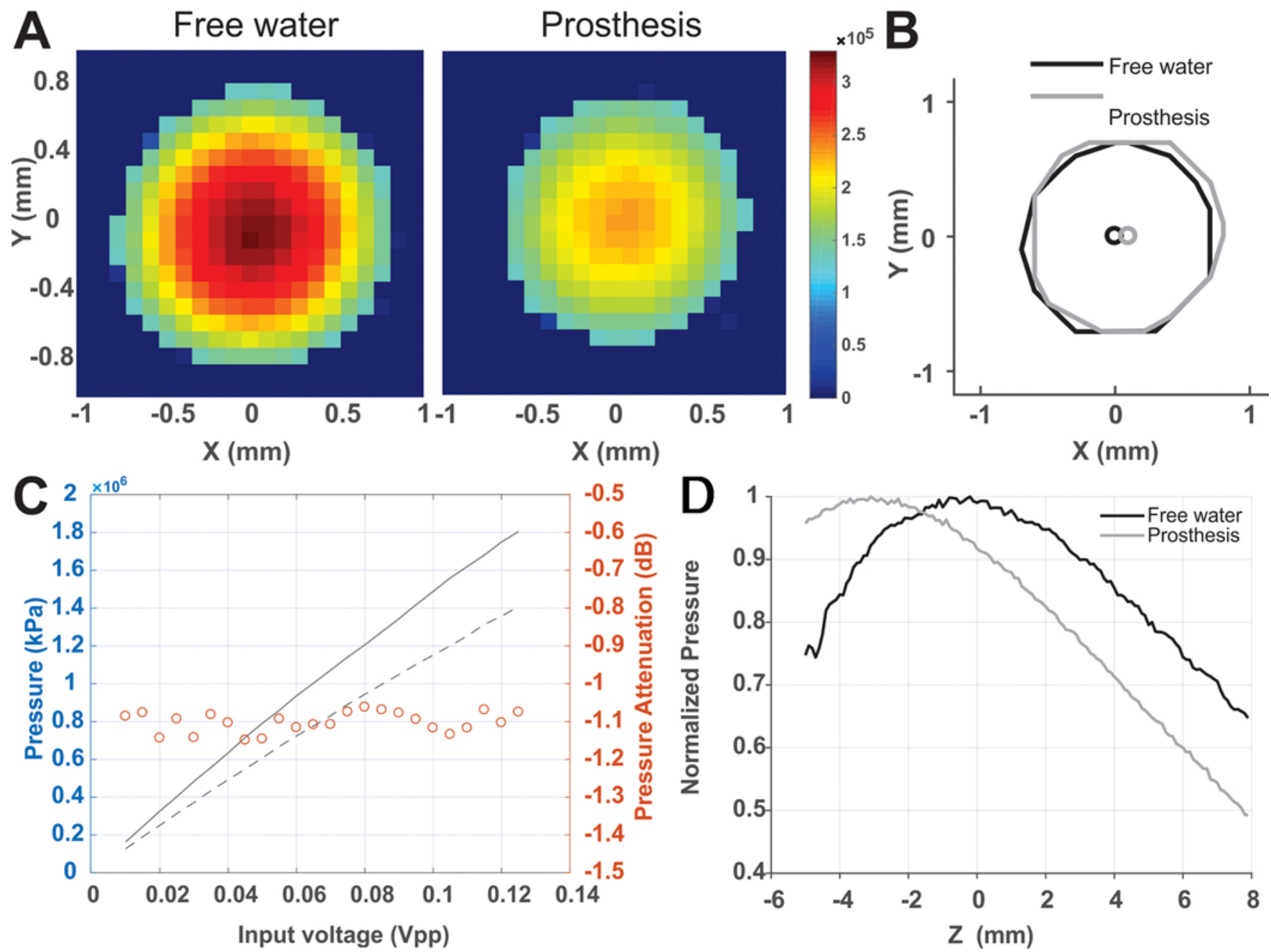

FIG. 6. Data for the 2.5-MHz transducer. A: XY cross-sectional pseudocolor pressure profiles at Z maximum. B: FWHM outline of the pressure map (large circles) and corresponding centroid (small circles). C: YY plot showing pressure in pascals (blue values) and attenuation through the prosthesis as compared to free water in decibels (red values) as a function of input voltage. The solid line is the recording in free water and the dashed line is the recording through the prosthesis. D: Pressure scan in Z access normalized to peak pressure. Zero on the $X$ axis denotes a pressure peak in the $Z$ plane found in free water. Negative values represent being closer to the transducer exit plane. Figure is available in color online only.

tenuation and adequate transmission of US energy through various combinations of US frequencies and transducers. Most notably, we tested transducers and frequencies that are used for preclinical focused US-based research applications and clinical neurosurgical practice. In an in vivo swine model, our prosthesis allowed for sonographic imaging of the intracranial space without image distortion, although a small degree of lateral attenuation was present in the most peripheral areas of the ultrasonic field. Nevertheless, this is a drastic improvement from what is expected when performing US imaging through the human skull..$^{12,15}$

The complex microarchitecture of the bones of the skull causes strong acoustic energy attenuation, which, in conjunction with the large discrepancies in acoustic impedances existing between the skin, the bone, and brain tissues, results in a drastic reduction of the transmission of ultrasonic energy propagating transcranially. ${ }^{3,15,35}$ Attenuation is mostly due to absorption and scattering of ultrasonic waves. ${ }^{12,15}$ Scattering is particularly relevant in the network of pores and trabeculae of the skull and plays the most important role in impeding transcranial US transmission, especially for beam frequencies used in diagnostic sonographic imaging (because the typical size of the trabecular structures of the diploe is $1 \mathrm{~mm}$, this heterogeneity of the bony structure becomes more relevant for frequencies higher than $1 \mathrm{MHz}$ ). This effect is due to the inhomogeneity of the cancellous bone of the skull, as small regions with different acoustic properties from surrounding areas tend to scatter the incident waves in all directions, resulting in a loss of acoustic intensity. Conversely, absorption, which mostly takes place in the cortical bone, has a lesser 

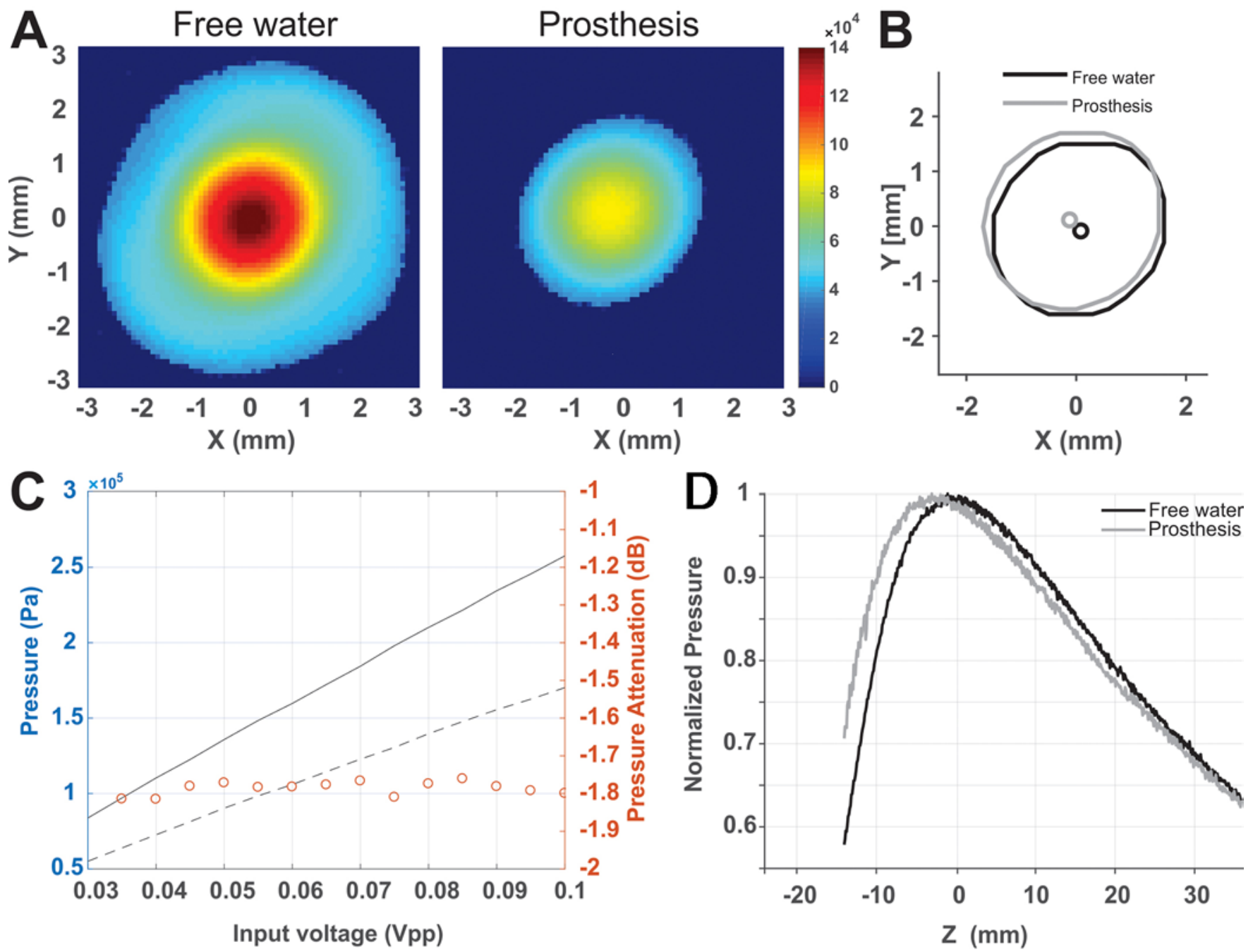

FIG. 7. Data for the 5-MHz transducer. A: XY cross-sectional pseudocolor pressure profiles at $Z$ maximum. B: FWHM outline of the pressure map (large circles) and corresponding centroid (small circles). C: YY plot showing pressure in pascals (blue values) and attenuation through the prosthesis as compared to free water in decibels (red values) as a function of input voltage. The solid line is the recording in free water and the dashed line is the recording through the prosthesis. D: Pressure scan in Z access normalized to peak pressure. Zero on the $X$ axis denotes a pressure peak in the $Z$ plane found in free water. Figure is available in color online only.

impact on attenuation of the US beams traversing the human skull..$^{15,35}$

In order to avoid the attenuation of US beams caused by the skull, and to subsequently improve the diagnostic and therapeutic possibilities of transcranial US, the cranial prosthesis that we developed is composed of a dense, low-porosity polymer with acoustic properties favorable for this purpose. Additionally, it has a homogeneous artificial structure and therefore a predictable interaction with acoustic beams along its entire surface.

We observed that increasing the US frequency augmented the degree of acoustic power loss, although this attenuation was still low compared to that caused by native cranial bone, which is known from in vitro studies to be high. ${ }^{12}$ Importantly, this loss was linear for all transducers tested with increasing input voltage. Even with this effect, when the highest frequency was tested, the US energy de- livered was sufficient for accurate imaging of the brain and is sufficient for low-intensity focused US-based research and clinical applications. ${ }^{25,26}$

In contrast to what is seen with the human skull, we noted that the degradation and distortion of the US beams passing through the prosthesis were minimal. ${ }^{8}$ The amount of shift of the US beams caused by the prosthesis was approximately $1 \mathrm{~mm}$ in the 3 planes that were examined. This degree of accuracy remained consistent across all transducers of different frequencies used in the experiment, which is demonstrated in Figs. 3-6. Since we used a low-end US probe, imaging quality was not optimal, and the sectional plane was not identical; however, the images acquired in vivo demonstrate little spatial distortion from the prosthesis and provide anatomical details and spatial relationships similar to those acquired through the dura (Fig. 7).

The technique of replacing the skull with materials 
Prada et al.
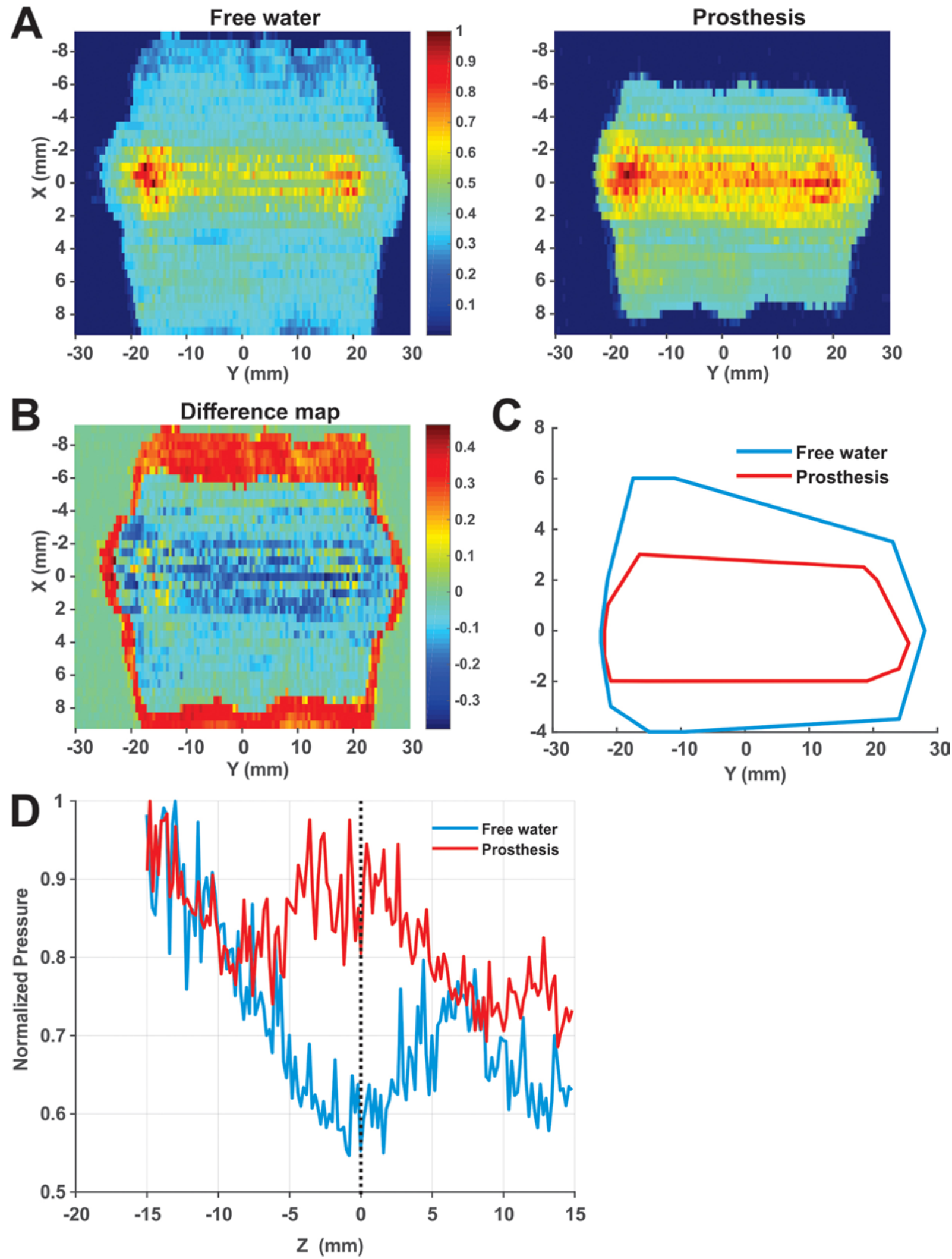

FIG. 8. Data for imaging transducer. A: XY cross-sectional pseudocolor normalized pressure profiles at $Z$ maximum. B: Difference map (free water minus prosthesis) of images in panel A. C: FWHM outline of the pressure map. D: Pressure scan in Z access normalized to peak pressure. Zero on the $X$ axis denotes a pressure peak in the $Z$ plane found in free water. Negative values represent being closer to the transducer exit plane. Figure is available in color online only. 
that are more permeable to US is not new. However, in the past, this technique was mostly used to deliver US beams to the brain with a therapeutic intent, rather than to obtain images for diagnostic and monitoring purposes. Various materials have been proposed to serve as cranial acoustic windows, mainly plastics and ceramics. ${ }^{4,6,14,34,43}$ The most comprehensive effort in this sense was performed in 1987 by Tobias and colleagues, ${ }^{43}$ who studied the acoustic properties of different biocompatible materials in vitro in order to deliver focused US to the brain to attain localized hyperthermia. These authors considered various properties of the prosthetic material, including the amount of energy attenuation when transmitting US transcranially; the spatial distortion of the US field and translation of its focal point; the mechanical stability of the materials; and the temperature changes occurring in the materials. Among the 4 different plastics analyzed, including high-density polyethylene, acrylic, polystyrene, and a commercial polymethyl methacrylate (PMMA), the material that performed best in transmitting US was highdensity polyethylene. This material transmitted the highest percentage of incident power, did not reach dangerous temperatures with the delivery of ultrasonic energy through it, did not overheat the underlying brain, and did not translate the US focus. On the contrary, commercially available PMMA allowed for the passage of the least amount of incident acoustic power (88\% energy transmitted through a 1-mm-thick polyethylene prosthesis vs $44 \%$ through a PMMA-based commercial cranioplasty material), thus ranking the worst material for a cranial acoustic window. ${ }^{43}$

Another approach for the transcranial delivery of acoustic energy to the brain has been to directly implant small, unfocused US transducers into the skull. ${ }^{6}$ This device avoids the obstacles posed by the bone in transmitting US, and it has been used in synergy with microbubble injection to transiently open the blood-brain barrier (BBB) in and around brain tumors in order to enhance the uptake of chemotherapeutic drugs by neoplastic cells. ${ }^{6}$ This system for disrupting the BBB has been demonstrated to be feasible and safe in experimental animal models and recently also in humans harboring cerebral high-grade gliomas. ${ }^{19,20}$ However, it is designed exclusively for therapeutic purposes and does not offer any possibility to image the brain.

More recently, Gutierrez et al. proposed the use of a pore-free transparent nanocrystalline $8-\mathrm{mol} \%$ yttria-stabilized zirconia ceramic implant to function as an optical/ structural cranial implant and serve as a cranial window to the brain. They tested prostheses of different densities and porosities, demonstrating that fully dense prostheses (with the lowest-porosity ceramic) transmitted US energy more efficiently. This is in agreement with our choice to implant a material with minimal porosity. ${ }^{15}$

Finally, some authors have attempted to image the brain with US through commercially available cranioplasties made of polyether ether ketone ${ }^{34}$ or PMMA. ${ }^{4}$ Preliminary results showed that these materials have greater US permeability than the native cranial bone and may allow for diagnostic US in order to identify intracranial anatomical structures (although with low resolution). However, an in- depth analysis of the acoustic properties of these materials has not been conducted yet.

All of the aforementioned studies provide a glimpse of the possible future applications of implantable cranial acoustic windows in neurosurgery. These implants make it possible to image the intracranial space with a fast, easy-to-use, and relatively inexpensive technology. More advantageously, these examinations could be performed at the bedside, as sonography obviates the need for patient transportation. Costs may also be reduced as expensive imaging procedures, such as CT and MRI, may be avoided when US is an option. Of note, as shown by intraoperative imaging studies, sonographic imaging has a spatial resolution on par with that of MRI, if a cranial window is present ${ }^{39,40}$ However, these studies are limited to an intraoperative examination, as they are performed through a temporary acoustic window. On the other hand, this prosthesis could be used as a permanent cranial window through which the patient's intracranial space is monitored. In this setting, US could be used to quickly and conveniently make specific clinical determinations, such as the efficacy of adjuvant therapies following tumor removal, ventricular size variation in patients with hydrocephalus, and cerebral blood vessel anatomy and flow. ${ }^{37}$ These types of acoustic windows could also be used, for example, to measure the response of intracranial tumors to antiangiogenetic and antivascular drugs when using US. This approach, referred to as contrast-enhanced US, consists of a new functional technique that enables a quantitative assessment of tumor perfusion using a mathematical model to analyze raw linear US data. Contrast-enhanced US has already been added to dedicated guidelines for the assessment of response to treatment in solid tumors (the European Federation of Societies for Ultrasound in Medicine and Biology guidelines on the clinical practice of contrast-enhanced US in 2011-2017 and the World Federation for Ultrasound in Medicine and Biology guidelines in $2012^{7,36,41}$ ), and, with the aid of our prosthesis, it could be extended to the assessment of the response to treatment of brain tumors..$^{23,24}$

From a therapeutic standpoint, US has been used for generating therapeutic lesions within maladaptive cerebral circuits, ${ }^{5,11,22}$ ablating brain tumors, ${ }^{29,30}$ opening the BBB to enhance drug delivery, ${ }^{17}$ dissolving emboli during cerebral infarction, ${ }^{32}$ and sensitizing tumor cells to radiotherapy and chemotherapy, ${ }^{45}$ as well as for transient low-intensity US-based neuromodulation. ${ }^{1,25,26}$ There are several additional novel indications for therapeutic US that are still emerging. ${ }^{18,38}$ However, as for imaging purposes, the main limitation for the delivery of US into the brain for ablation or other therapeutic applications is the skull, especially when high intensities are required, such as for thermal ablation. ${ }^{18,38}$ At first, the bone was removed for single-application, high-intensity therapies. ${ }^{16,21}$ Thereafter, the development of special hemispheric phased-array transducers coupled with MRI guidance and rigid skull fixation has allowed for the delivery of focused US energy through the intact skull without damaging the intervening brain parenchyma or overheating the skull. Despite its completely external nature, however, this system has several limitations. These include the small dimension of the 
focus, which makes the ablation of large volumes of tissue difficult and time consuming, and the ability to effectively treat only centrally located intracranial regions (where US can be focused optimally). The currently proposed solutions to overcome these issues consist of enhancing the effect of US with externally delivered agents, such as preformed microbubbles, ${ }^{31}$ inserting probes interstitially, ${ }^{13}$ or using very short pulses to fractionate and liquefy tissue volumes. ${ }^{42}$ An implanted cranial window would allow for the repeated delivery of focused and unfocused US beams with little attenuation and direct, real-time US guidance for ablative or therapeutic applications without the need for large hemispheric transducers, interstitial probes, or agents that enhance the action of US. An acoustic window would also allow the delivery of sufficient energy for the ablation of peripherally located intracranial regions. This would result in an extension of the "treatment envelope" of the currently available devices for MRI-guided focused US. ${ }^{10}$

\section{Limitations}

In our study, we did not evaluate the effect of prosthesis thickness on US transmission. Also, we did not assess thermal changes occurring within the prosthesis. Overheating could indeed lead to damage of the underlying meninges and cerebral cortex. In addition, we did not evaluate the ability of the prosthesis to protect the brain from direct force. However, polyethylene-based prostheses are able to withstand most common impacts that a person may encounter, and the strength of this material is witnessed by its routine use in orthopedic implants. ${ }^{2,43}$ We did not assess imaging through the prosthesis with the skin closed. The skin is known to be very permeable to US, as demonstrated by multiple thoracic and abdominal sonographic imaging studies performed over several decades. ${ }^{44}$

Further studies will need to be performed by taking these details into account. Subsequent investigations will also have to assess specific ultrasonography applications through the prosthesis in preclinical models, including imaging and therapeutic low-intensity and high-intensity US-based applications (BBB opening, contrast ultrasonography, vascular micro-Doppler, neuromodulation, and focused US thermal ablation). Imaging will also have to be performed with high-end US instruments to better determine the detail of sonographic images that can be obtained when imaging through the prosthesis, as well as more advanced imaging modalities such as contrast-enhanced US and elastography. ${ }^{9}$ Finally, the prosthesis will have to be tested in human subjects.

\section{Conclusions}

Our cranial prosthesis has been shown to be a valid medium for the intracranial delivery of both focused and unfocused US and for imaging the intracranial space with US. Importantly, we studied the same transducers and US parameters that are currently used for diagnostic and therapeutic neurosurgical applications. Transmission of US energy through the prosthesis was successfully performed with a significantly lower energy attenuation than that reported to occur through the native cranial bone. In addition, focused delivery of US through the prosthesis was accurate both in vitro and in vivo. These results were consistent across all different frequencies and transducers used, suggesting that the prosthesis could be used in several diagnostic and therapeutic US-based applications, including bedside imaging of the brain and focused US ablation or neuromodulation.

\section{Acknowledgments}

This work was supported by University of Virginia Health Sciences Center, Charlottesville, Virginia.

\section{References}

1. Ai L, Bansal P, Mueller JK, Legon W: Effects of transcranial focused ultrasound on human primary motor cortex using $7 \mathrm{~T}$ fMRI: a pilot study. BMC Neurosci 19:56, 2018

2. Amstutz HC: Biomaterials for artificial joints. Orthop Clin North Am 4:235-248, 1973

3. Aubry JF, Tanter M: MR-guided transcranial focused ultrasound. Adv Exp Med Biol 880:97-111, 2016

4. Belzberg M, Shalom NB, Yuhanna E, Manbachi A, Tekes A, Huang J, et al: Sonolucent cranial implants: cadaveric study and clinical findings supporting diagnostic and therapeutic transcranioplasty ultrasound. J Craniofac Surg 30:14561461,2019

5. Bond AE, Shah BB, Huss DS, Dallapiazza RF, Warren A, Harrison MB, et al: Safety and efficacy of focused ultrasound thalamotomy for patients with medication-refractory, tremordominant Parkinson disease: a randomized clinical trial. JAMA Neurol 74:1412-1418, 2017

6. Carpentier A, Canney M, Vignot A, Reina V, Beccaria K, Horodyckid C, et al: Clinical trial of blood-brain barrier disruption by pulsed ultrasound. Sci Transl Med 8:343re2, 2016

7. Claudon M, Dietrich CF, Choi BI, Cosgrove DO, Kudo M, Nolsøe CP, et al: Guidelines and good clinical practice recommendations for contrast enhanced ultrasound (CEUS) in the liver-update 2012: A WFUMB-EFSUMB initiative in cooperation with representatives of AFSUMB, AIUM, ASUM, FLAUS and ICUS. Ultrasound Med Biol 39:187210,2013

8. Clement GT, Sun J, Giesecke T, Hynynen K: A hemisphere array for non-invasive ultrasound brain therapy and surgery. Phys Med Biol 45:3707-3719, 2000

9. Del Bene M, Perin A, Casali C, Legnani F, Saladino A, Mattei L, et al: Advanced ultrasound imaging in glioma surgery: beyond gray-scale B-mode. Front Oncol 8:576, 2018

10. Eames MD, Farnum M, Khaled M, Elias WJ, Hananel A, Snell JW, et al: Head phantoms for transcranial focused ultrasound. Med Phys 42:1518-1527, 2015

11. Elias WJ: A trial of focused ultrasound thalamotomy for essential tremor. N Engl J Med 375:2202-2203, 2016

12. Fry FJ, Barger JE: Acoustical properties of the human skull. J Acoust Soc Am 63:1576-1590, 1978

13. Ghoshal G, Gee L, Heffter T, Williams E, Bromfield C, Rund L, et al: A minimally invasive catheter-based ultrasound technology for therapeutic interventions in brain: initial preclinical studies. Neurosurg Focus 44(2):E13, 2018

14. Gordon CR, Santiago GF, Huang J, Bergey GK, Liu S, Armand M, et al: First in-human experience with complete integration of neuromodulation device within a customized cranial implant. Oper Neurosurg (Hagerstown) 15:39-45, 2018

15. Gutierrez MI, Penilla EH, Leija L, Vera A, Garay JE, Aguilar G: Novel cranial implants of yttria-stabilized zirconia as acoustic windows for ultrasonic brain therapy. Adv Healthc Mater 6:6, 2017 
16. Heimburger RF: Ultrasound augmentation of central nervous system tumor therapy. Indiana Med 78:469-476, 1985

17. Hernot S, Klibanov AL: Microbubbles in ultrasound-triggered drug and gene delivery. Adv Drug Deliv Rev 60:11531166,2008

18. Hersh DS, Kim AJ, Winkles JA, Eisenberg HM, Woodworth GF, Frenkel V: Emerging applications of therapeutic ultrasound in neuro-oncology: moving beyond tumor ablation. Neurosurgery 79:643-654, 2016

19. Horodyckid C, Canney M, Vignot A, Boisgard R, Drier A, Huberfeld G, et al: Safe long-term repeated disruption of the blood-brain barrier using an implantable ultrasound device: a multiparametric study in a primate model. J Neurosurg 126:1351-1361, 2017

20. Idbaih A, Canney M, Belin L, Desseaux C, Vignot A, Bouchoux G, et al: Safety and feasibility of repeated and transient blood-brain barrier disruption by pulsed ultrasound in patients with recurrent glioblastoma. Clin Cancer Res 25:3793-3801, 2019

21. Jagannathan J, Sanghvi NT, Crum LA, Yen CP, Medel R, Dumont AS, et al: High-intensity focused ultrasound surgery of the brain: part 1-A historical perspective with modern applications. Neurosurgery 64:201-211, 2009

22. Jung HH, Kim SJ, Roh D, Chang JG, Chang WS, Kweon EJ, et al: Bilateral thermal capsulotomy with MR-guided focused ultrasound for patients with treatment-refractory obsessivecompulsive disorder: a proof-of-concept study. Mol Psychiatry 20:1205-1211, 2015

23. Lassau N, Bonastre J, Kind M, Vilgrain V, Lacroix J, Cuinet $\mathrm{M}$, et al: Validation of dynamic contrast-enhanced ultrasound in predicting outcomes of antiangiogenic therapy for solid tumors: the French multicenter support for innovative and expensive techniques study. Invest Radiol 49:794-800, 2014

24. Lassau N, Chami L, Chebil M, Benatsou B, Bidault S, Girard E, et al: Dynamic contrast-enhanced ultrasonography (DCEUS) and anti-angiogenic treatments. Discov Med 11:18-24, 2011

25. Legon W, Ai L, Bansal P, Mueller JK: Neuromodulation with single-element transcranial focused ultrasound in human thalamus. Hum Brain Mapp 39:1995-2006, 2018

26. Legon W, Bansal P, Tyshynsky R, Ai L, Mueller JK: Transcranial focused ultrasound neuromodulation of the human primary motor cortex. Sci Rep 8:10007, 2018

27. Legon W, Sato TF, Opitz A, Mueller J, Barbour A, Williams A, et al: Transcranial focused ultrasound modulates the activity of primary somatosensory cortex in humans. Nat Neurosci $17: 322-329,2014$

28. Lind NM, Moustgaard A, Jelsing J, Vajta G, Cumming P, Hansen AK: The use of pigs in neuroscience: modeling brain disorders. Neurosci Biobehav Rev 31:728-751, 2007

29. Mauri G, Nicosia L, Xu Z, Di Pietro S, Monfardini L, Bonomo G, et al: Focused ultrasound: tumour ablation and its potential to enhance immunological therapy to cancer. Br J Radiol 91:20170641, 2018

30. McDannold N, Clement GT, Black P, Jolesz F, Hynynen K: Transcranial magnetic resonance imaging-guided focused ultrasound surgery of brain tumors: initial findings in 3 patients. Neurosurgery 66:323-332, 2010

31. McDannold N, Zhang YZ, Power C, Jolesz F, Vykhodtseva $\mathrm{N}$ : Nonthermal ablation with microbubble-enhanced focused ultrasound close to the optic tract without affecting nerve function. J Neurosurg 119:1208-1220, 2013

32. Monteith SJ, Kassell NF, Goren O, Harnof S: Transcranial MR-guided focused ultrasound sonothrombolysis in the treatment of intracerebral hemorrhage. Neurosurg Focus 34(5):E14, 2013

33. Mueller JK, Ai L, Bansal P, Legon W: Computational exploration of wave propagation and heating from transcrani- al focused ultrasound for neuromodulation. J Neural Eng 13:056002, 2016

34. Mursch K, Behnke-Mursch J: Polyether ether ketone cranioplasties are permeable to diagnostic ultrasound. World Neurosurg 117:142-143, 2018

35. Pinton G, Aubry JF, Bossy E, Muller M, Pernot M, Tanter M: Attenuation, scattering, and absorption of ultrasound in the skull bone. Med Phys 39:299-307, 2012

36. Piscaglia F, Nolsøe C, Dietrich CF, Cosgrove DO, Gilja OH, Bachmann Nielsen M, et al: The EFSUMB guidelines and recommendations on the clinical practice of contrast enhanced ultrasound (CEUS): update 2011 on non-hepatic applications. Ultraschall Med 33:33-59, 2012

37. Prada F, Del Bene M, Saini M, Ferroli P, DiMeco F: Intraoperative cerebral angiosonography with ultrasound contrast agents: how I do it. Acta Neurochir (Wien) 157:1025-1029, 2015

38. Prada F, Kalani MYS, Yagmurlu K, Norat P, Del Bene M, DiMeco F, et al: Applications of focused ultrasound in cerebrovascular diseases and brain tumors. Neurotherapeutics 16:67-87, 2019

39. Prada F, Perin A, Martegani A, Aiani L, Solbiati L, Lamperti $\mathrm{M}$, et al: Intraoperative contrast-enhanced ultrasound for brain tumor surgery. Neurosurgery 74:542-552, 2014

40. Prada F, Vitale V, Del Bene M, Boffano C, Sconfienza LM, Pinzi V, et al: Contrast-enhanced MR imaging versus contrast-enhanced US: a comparison in glioblastoma surgery by using intraoperative fusion imaging. Radiology 285:242249, 2017

41. Sidhu PS, Cantisani V, Dietrich CF, Gilja OH, Saftoiu A, Bartels E, et al: The EFSUMB guidelines and recommendations for the clinical practice of contrast-enhanced ultrasound (CEUS) in non-hepatic applications: update 2017 (long version). Ultraschall Med 39:e2-e44, 2018

42. Sukovich JR, Cain CA, Pandey AS, Chaudhary N, CameloPiragua S, Allen SP, et al: In vivo histotripsy brain treatment. J Neurosurg 131:1331-1338, 2019

43. Tobias J, Hynynen K, Roemer R, Guthkelch AN, Fleischer AS, Shively J: An ultrasound window to perform scanned, focused ultrasound hyperthermia treatments of brain tumors. Med Phys 14:228-234, 1987

44. Wells PN, Liang HD: Medical ultrasound: imaging of soft tissue strain and elasticity. J R Soc Interface 8:1521-1549, 2011

45. Yu T, Li SL, Zhao JZ, Mason TJ: Ultrasound: a chemotherapy sensitizer. Technol Cancer Res Treat 5:51-60, 2006

\section{Disclosures}

F. Prada and F.D.M. are listed as coinventors on patents related to the production of the prosthesis used in this study, which is owned by In.Tra. F. Prada, F.D.M., and L.S. own stock in In.Tra. In.Tra. has provided the prosthesis for preclinical use.

\section{Author Contributions}

Conception and design: Prada, Padilla, Solbiati, DiMeco, Legon. Acquisition of data: Prada, Franzini, Legon. Analysis and interpretation of data: Prada, Franzini, Moosa, Legon. Drafting the article: Franzini, Legon. Critically revising the article: all authors. Reviewed submitted version of manuscript: Prada, Franzini, Moosa, Padilla, Moore, Solbiati, Legon. Approved the final version of the manuscript on behalf of all authors: Prada. Statistical analysis: Legon. Administrative/technical/material support: Prada, Legon. Study supervision: Prada, Legon.

\section{Correspondence}

Francesco Prada: Fondazione IRCCS Istituto Neurologico C. Besta, Milan, Italy. francesco.prada@istituto-besta.it. 